\title{
Individual Differences as a Crucible in Theory Construction
}

\author{
BENTON J. UNDERWOOD N.orthwestern University
}

My proposal is that we should formulate our nomothetic theories in a way that will allow an immediate individual-differences test. I am proposing this because, among other benefits, I believe this approach will make individual differences a crucible in theory construction. The argument I advance is applicable to theory construction in all areas of experimental psychology, but my illustrations come largely from the areas of learning and memory. I feel impelled initially to reconstruct as best $I$ can the reasons that led me to compose an article dealing with theory construction. It has resulted from a professional uneasiness that has grown over the past few years. These pinpricks of uneasiness seemed to say that our profession needed to open a discussion of theory construction in psychology, a discussion led by psychologists, for psychologists. When the uneasy feelings were articulated in this manner, I was able to identify three developments that had been responsible for the pinpricks. And then a fourth development took place which led me to presume I might have something to say that could just possibly initiate the discussion.

The first source of uneasiness was quite an unlikely one, namely, the undergraduate student. On occasion, a perceptive one will ask me, "How do you get a theory?" How does one answer this question? I found myself answering with a few pieces of trivia of the kind that any experienced teacher has ready for such moments. My lack of

This article was a Distinguished Scientific Contribution Award address presented at the meeting of the American Psychological Association, New Orleans, August 1974. It was supported by the Psychological Sciences Division, Office of Naval Research under Contract N00014-67-A-0356-0010, Contract Authority Identification NR 154-321. Reproduction in whole or in part is permitted for any purpose of the U.S. Government.

Requests for reprints should be sent to Benton J. Underwood, Department of Psychology, Northwestern University, Evanston, Illịnois 60201. a guiding answer was demonstrated most blatantly when I found myself turning to anecdotes to shunt the question aside. Thus, I would tell the student that one great theoretical insight in the history of science is alleged to have occurred during a bath. So, perhaps, taking a bath would be a way to get a theory. But, of course (I told him), if you really want to develop a majestic theory, the only avenue open is to learn to play the violin and go to Princeton.

The question remained unanswered, but I did set about to see if I could put something down on paper of a systematic nature that might be given to a student who would be so brazen as to ask such a question. To some extent, what I say here was prepared for such a student.

The second stimulus I can identify as being involved in my uneasiness relates to developments in the area of memory, including the offshoot now called information processing. In particular, I refer to structural model building. Certainly, in the last dozen years, the favorite after-class occupation of many college professors has been that of building models of memory. Just what is responsible for this furious activity is not entirely apparent to me. One might guess that the flow diagram presented by our English colleague, Donald Broadbent, in his 1958 book Perception and Communication was involved, but I choose not to saddle him with this reverse lend-lease if he doesn't want to take the responsibility for it. The fact remains that we have models running out of our ears, and there seems to be no surcease.

This may be quite healthy; at least lots of people are getting skilled in drawing boxes, arrows, and circular nodes. But all of these models cannot be right, or even useful or believable, and evaluation seems to be rather low on the priority list. It seems to be easier to formulate a new model than to test an old one, and one never gets pinned down that way. I am being unfair, of course, and this 
is particularly troublesome because the model builders are very friendly people; many are my friends. All I ask of the builders is please, sooner or later, come up for breath and see what you have wrought. Is this really the way we want theory development to occur?

A third stimulus was a book published in 1967 called Learning and Individual Differences. It represents the thinking of a number of investigators brought together by Robert Gagné for a conference at the University of Pittsburgh. Reading this book gave me a small intellectual abrasion that has continued to fester over the years. I was unable to get rid of it by saying to myself that the problem of individual differences is someone else's responsibility. I finally came to accept the notion that individual differences ought to be considered central in theory construction, not peripheral. How can we make individual differences of central focus in our theories? This volume contains ideas, and I suspect that if one were to juxtapose what was said at that conference with some of my later comments, the similarity would be found to be more than coincidental.

And finally, certain events which occurred in our laboratory served as the catalyst for the final step, namely, that of trying to bring individual differences into the mainstream of theory construction. Some background is necessary. In 1966 an article was published (Ekstrand, Wallace, \& Underwood) which proposed that verbal-discrimination learning (which is a special kind of a recognition task) is mediated by the subject discriminating the apparent frequency differences between the right and wrong words in each pair. The idea was subsequently extended to the more classical recognition procedures. The theory, commonly called frequency theory, has had some success in predicting the consequences of manipulating a number of independent variables. A severe critic might argue about the use of the word "success," but that is unimportant for the present paper. But it is important to understand the basic nature of the theory.

As I understand the strict use of the term model, it means that a set of empirical relationships developed in one area of discourse is applied to another area of research as a possible explanatory system. As an extreme case, if the laws and relationships among the functions of the organs of the digestive system were applied to memory as an explanatory system, this would be an illustration of the true meaning of the word model. This transfer from one area to another need not be across disciplines; it can be within a discipline. If the laws of learning are used to try to account for bizarre behaviors, it would be a form of modeling. Frequency theory, in the language of modeling, is a within-discipline model of an unusual kind. The theory asserts that the laws and relationships that hold for frequency discrimination (as viewed, perhaps, in the classical psychophysical sense) will determine the performance in the usual recognitionmemory study. The unusual nature of the theory lies in the fact that when the theory was formulated there wasn't a body of laws and relationships concerning frequency discriminations. It has been necessary, therefore, to develop both areas simultaneously. Nevertheless, the theory is quite explicit on the central point; the facts of frequency discrimination must hold for the recognition situation or the theory is in trouble.

The theory as stated is a nomothetic theory, since the thinking was geared entirely to mean performances and mean frequency discriminations. This form of thinking can be blinding. But finally (and there were a number of developments that were responsible but that will not be set down here) the time came when a now obvious implication forced itself into our thinking. The whole fabric of the theory, quite by accident, rests on a postulate that is in fact an individual-differences postulate. However we might have stirred uncomfortably when we realized this and however we might have tried to find some escape, the implication would not go away. A subject who demonstrated fine or precise frequency discriminations must show good recognition memory; a subject who demonstrated poor or imprecise frequency discriminations must show poor recognition memory. What a devastating relationship to contemplate so late in the development of the theory, particularly so since we knew that both frequency discriminations and recognition memory yielded quite reliable scores. In this case, when the belated tests were made, the outcomes showed the prerequisite relationships. But suppose this had not been the outcome? Suppose we had found a zero correlation between measures of frequency discrimination and measures of recognition memory? The theory would simply have to be dropped. That we had demonstrated the necessary relationships on the fiftieth study, was, perhaps, a stroke of luck. The point is that, had we been so wise as to perceive it, the fiftieth study 
should have been the first study. If the data from this first study did not approve of the individualdifferences relationship inherent in the theory, there would have been no theory, no 50 studies.

Let me now state the generalized case. If we include in our nomothetic theories a process or mechanism that can be measured reliably outside of the situation for which it is serving its theoretical purpose, we have an immediate test of the validity of the theoretical formulation, at least a test of this aspect of the formulation. The assumed theoretical process will necessarily have a tie with performance which reflects (in theory) the magnitude of the process. Individuals will vary in the amount of this characteristic or skill they "possess." A prediction concerning differences in the performance of the individuals must follow. A test of this prediction can yield two outcomes. If the correlation is substantial, the theory has a go-ahead signal, that and no more; the usual positive correlations across subjects on various skills and aptitudes allow no conclusion concerning the validity of the theory per se. If the relationship between the individualdifferences measurements and the performance is essentially zero, there is no alternative but to drop the line of theoretical thinking. It is this form of reasoning that has led to the title of this article; individual differences may indeed be used as a crucible in nomothetic theory construction. The approach, I believe, provides a critical test of theories as they are being born; if they fail to pass the test, they should neither see the light of day nor the pages of the Psychological Review.

I now must turn to a broader perspective of theory construction and show how the individualdifferences approach fits into this perspective. In effect, I am going to try to give the student an answer to his inquiry, albeit an indirect and an incomplete one. What I want to tell the student. is that there seems to be a common way in which theoretical thinking gets started, and then I will provide him with some guidelines with which he should be concerned if he wants his theory to be disciplined in the sense that it can be discredited as well as affirmed.

A behavioral phenomenon is defined by the relationship between some independent variable and measured behavior. As research develops, certain key phenomena are identified and a body of empirical knowledge builds up around them. Thus, we have such key phenomena as extinction, retroactive inhibition, altruism, motivation, space per- ception, and so on. Now, even in the definition of such phenomena we may tend to allow an internalization of a process. It is not a great leap to recognize that the independent variable produces an influence only because it did something to the "workings" of the subject. When, over successive trials, we observe an increase in the number of correct responses given by a subject, we say that learning has occurred, although learning is neither the number of correct responses nor the trials. Learning is a term we use to represent the relationship between the two, and frequently also to represent the implicit belief that something has "gone on" in the subject. It is almost impossible to think of a term such as motivation without thinking of it at the same time as being changes in the organism.

The above illustrations suggest that it is difficult to avoid thinking about intervening processes even when thinking about the definition of so-called empirical phenomena. Theoretical efforts merely make the thinking about intervening processes more formal and more deliberate. The basis of theorizing is that of proposing intervening processes (some prefer the term mechanisms) that will mediate the observed empirical relationships between various independent variables and the key phenomenon of interest. I will not entertain the question of whether we should or should not be theorizing; not many can avoid it. But it is reasonable to ask what we propose to achieve by a theory. From one point of view, theorizing is simply one of the later steps along the chain of steps known as data reduction. We try to comprehend the scores of 100 subjects by getting a statistical description of the scores. We try to comprehend the scores of five groups given different levels of an independent variable by plotting the mean scores above the five levels of the variable. We try to summarize a number of different experiments in an area by trying to extract the commonalities and stating the empirical generalizations. We try, by theorizing, to state basic processes that could underlie the behavior and produce the several laws within the area of interest. Theorizing is always reductive in the sense that we try to propose processes more elementary (but more general) or basic than the phenomena for which we are trying to account. In all of the steps of data reduction, including theoretical speculation, we are trying to produce the ultimate in economy of thought.

Now (still speaking to the student), what guide- 
lines can be used in proposing the intervening process? There are many obvious ones, such as explicitness and testability, but these are generally necessary consequences of others. I propose three guidelines.

The first guideline is a compound one: The theory must assume at least two intervening processes, and these processes must interact in some way to relate the independent variables to the dependent variable. This statement needs to be unpacked. Why must we have two processes? A single-process theory must always be isomorphic to empirical relationships. If $I$ assume that interference as a theoretical process is responsible for forgetting, assuming that and no more, the empirical relationships give the complete story, since interference must vary in magnitude as forgetting varies in magnitude. As a theoretical concept, it is superfluous and has no predictive power. It can also be seen that if two intervening processes are assumed, but which vary in magnitude in exactly the same way for all independent variables, it reduces essentially to a single-process theory.

The moment we propose two intervening processes that, for at least one independent variable, have different functions and hence interact, we begin to get predictive power. This guideline seems to have been followed for many years, as witness the many different theoretical approaches including an excitatory and an inhibitory process (by whatever names) which are assigned differential functions for certain independent variables. The interaction can be "inserted" at two different points. It is probably most common to provide the interaction by having different functional relationships between the two assumed processes and the independent variables. But it would be quite possible to have the theoretical relationships be the same for the independent variable but differ with regard to their influence on the dependent variable.

I emphasize the necessity of the interaction between the intervening processes for at least one independent variable because I do not believe it has been clearly enunciated in recent years. I emphasize it also for quite a different reason. Those of us who teach undergraduates know that teaching them how to see, verbalize, and become generally facile in thinking about empirical interactions in data know that it is adventurous, to say the least. But it seems necessary for them to develop this skill if we want them also to grasp the nature of predictive theory.
In the statement of this guideline it was indicated that there must be at least two intervening processes. The complexity of a theory increases directly as the number of postulated processes increase. Obviously we try to keep the number of processes to a minimum, but if it becomes necessary to add, we must add. In so doing we recognize that a problem in understanding will necessarily arise. Some idea of the magnitude of the problem of understanding can be obtained by trying to comprehend, for example, an empirical interaction among four variables. The complexity may be necessary and, if it is necessary, I believe we will find it imperative to represent the theoretical processes in strict mathematical terms so that the derivations can be unambiguous.

A second guideline I would suggest to my student is implicit in a number of previous statements. It is that any assumed process must be tied to at least one independent variable. I would point out to the student that not all would agree with this guideline, but also I would feel impelled to tell him that if he doesn't abide by this guideline he is likely to find himself in a pack of trouble. Nothing is more conducive to the infection of a theory by ploglies and homunculi than a freefloating intervening process. I read or heard (from a source that $I$ have not been able to identify) that the idea that an intervening process must be tied to at least one independent variable is no longer considered essential and should be abandoned as an unnecessary stricture on the imagination needed for theorizing. It seems to me that it doesn't take much imagination to realize that to abandon this rule is to invite chaos.

The third guideline is concerned with the nature of the intervening processes to be postulated. I think we must allow great latitude, perhaps along several different dimensions, in proposed intervening processes. At one extreme, they may be strictly abstract mathematical propositions that disclaim any correspondence or relationship to a psychological process with which we might identify intuitively. Although we might not be able to resonate personally to such abstract, impalpable processes, they do have the very distinct advantage of avoiding misinterpretations that may occur when common psychological terms are used for identifying the processes. In any event, some of my subsequent comments cannot, it will be seen, be germane to the completely abstract intervening process. 
At the other extreme, we may assume an intervening process that is more or less given by an empirical relationship in another area of psychological inquiry. Earlier I described the basic idea of frequency theory; it is a good illustration of this low-level form of theorizing. In between the two extremes there are various steps, and in fact many theories represent a mixed bag with regard to placement along the dimension of abstractness.

Now obviously, under this guideline, I have one specific proposal in mind, namely, that in choosing theoretical processes if at all possible choose at least one which has some possibility of yielding an individual-differences interpretation, as has been described. The illustration I gave from frequency theory may seem obvious and atypical. In fact, however, after I worked on the matter with other theoretical notions, I began to form the opinion that the individual-differences approach could in principle be implemented with any but the more abstract propositions. Let me give three illustrations.

If a free-recall list includes words occurring more than once, the recall of the words given spaced repetition will be superior to those given massed repetitions. Our theoretical thinking emphasized a reduced processing of the items when they were massed. Some indirect tests showed this to have some support. In thinking about this theory in light of the guideline under discussion, it seemed beyond doubt that subjects must differ in their propensity to attenuate processing. Now, if we could measure this tendency independently, and if it is a reliable individual-differences variable, we could make a test to tell us whether the theory should be dropped or whether we had a license to continue its development. Such a test now seems possible, and we will undertake it in the fall. My only regret is that we did not formulate this approach several years ago when the theory first came into being.

Assume that a theory is proposed for serial learning which includes a process identified as generalization along a spatial dimension. We have the techniques for measuring generalization outside the serial learning task. The role that generalization is assigned in serial learning must surely be in some way predictably different for subjects having different generalization gradients.

My skimming of abstracts has suggested that some investigators studying the skills involved very early in the process of learning to read are suggesting that the subject's ability to develop an internal schemata of each of the letters is important. The schemata will allow a "match" even though some distortion is present in the visually presented letters. At the same time, the schemata should not be too broad or it will accept wrong letters as a match. Would it be possible to get an independent measure of the characteristics of the schemata without visual inputs of letters? Adults can identify very accurately individual letters when "printed" on the skin of the back with the index finger (wielded by another person, of course). Would this be useful for measuring schemata in pure form in children? And, then, would this predict errors in identification of visually presented letters?

These three illustrations are sufficient to see the direction I think this approach might take. By this time, objections may be cropping up. I hope these can be anticipated in the three possible objections I will now discuss. Two of the objections can be handled quite satisfactorily, I believe; one is a little more difficult. We will start with the difficult one.

I used frequency theory to illustrate a basic application of the individual-differences approach. The theory, in addition to assuming that a frequency discrimination is critical, also assumes that the subject applies a rule to cover all pairs in a verbal-discrimination task, namely, the rule to choose the word with the highest apparent frequency in each pair. Suppose that frequency discrimination and the rapidity of rule discovery are correlated. That subjects with good frequency discrimination are also good verbal-discrimination learners might then be due to the fact that they learn rules quickly and that some rule other than the frequency rule is mediating the performance. In this particular case, there are several auxiliary facts (which will not be detailed here) that rule out this possibility, but it may not be possible to do this in other situations. The generalized issue concerns the correlative relationship between the performance produced by the intervening process being evaluated as an individual-differences variable, and the performance produced by other processes in the theory. The solution is to make sure that only the symptoms of the individual-differences variable are being measured in the case in which the other processes may also be operating to influence performance. If this cannot be done it may produce a positive conclusion concerning the 
individual-differences variable when in fact the evidence producing the positive conclusion results from a correlation with the consequences of the other theoretical process. To avoid this, we must in some way neutralize the effects of the other variable. Experimental ingenuity should find a way to accomplish this. But even if a solution is not found, it should be clear that we are no worse off than we are at present when this approach is not used. However, the most important function of the individual-differences approach is that of nipping an inappropriate theoretical notion in the bud, and this is indicated when a zero correlation is found. So, the first objection is by no means a lethal one.

The second objection is quite a different one. I think it a certainty that the individual-differences approach described here will be most applicable when the process used theoretically has more or less empirical status in another area of research within psychology. What constitutes another or different area? Behavior is behavior, some might say. I can illustrate the question in as stark a manner as possible. Suppose there is an empirical relationship derived from the learning of two-syllable words. Now, we say, we are going to use this relationship theoretically as a part of a theoretical system to explain the learning of three-syllable words. We go through the individual-difference routine and find a high positive correlation that, according to the argument which has been advanced, gives us license to proceed with the development of the theory. There isn't a good name for such thinking; around our laboratory we speak of this by the rather crude but descriptive word incest. We must be sure that when we use the approach I am advocating we are dealing with no more than kissin' cousins. I suppose that good judgment must be imposed, or that the union is acceptable when it is not intuitively obvious that they should be related. And further, we are always transferring what appears to be a simple process for use as a theoretical concept (along with the use of at least one other) in attempting to account for performance on a more complex task than the one used to measure the simple process directly.

A third objection that may be raised is not directed necessarily toward the individual-differences approach but toward the use of a relationship discovered in one area as an explanatory concept in another. It might be insisted that this approach doesn't explain anything. As an illustration: if frequency discrimination can be used to account for recognition memory, it is fine and good, but what has been gained? It merely means that to understand recognition memory, we must understand the processes involved in frequency discriminations. This objection is without validity and can be raised about any theoretical approach using behavioral constructs (as opposed to the use of physiological constructs). The whole idea behind behavioral theory is to reduce the number of independent processes to a minimum; to find that performance on two apparently diverse tasks is mediated at least in part by a single, more elementary, process is a step toward this long-range goal.

There is one further point that should be made, relative to the third guideline, the discussion of which has largely consisted of trumpets being blown in support of the individual-differences approach. There is nothing in this approach that prevents the use of mathematical expressions for the theoretical processes. Indeed, they should be used by all who have the skills and the wills. All that is being proposed is that when possible, one of the theoretical processes be identified in such a way that it is at least remotely feasible that it could be measured as an individual-differences variable.

It should be apparent that the more traditional attempts to relate nomothetic theories to individual differences by using standardized tests, for example, paper-and-pencil tests, are quite in line with the approach proposed here. Thus, tests of manifest anxiety, introversion-extroversion, ego strength, and so on, have been used to identify individual differences that are in turn said to be identifiable with assumed processes in nomothetic theories. If there are differences in the approaches, they lie in the indirectness of the measurements and the types of conclusions drawn when the theoretical tests are made. A positive correlation is to be responded to in the way discussed earlier. A zero correlation, however, is frequently not used in a critical, decisive manner. The investigator far too frequently puts the blame on the paperand-pencil test for not measuring what it is said to measure, rather than putting the blame on the assumed theoretical relationship. Under these circumstances, the individual-differences variable is not a crucible in theory construction. Rather, it 
is an interesting adjunct of theoretical development.

As a final point, I would like to suggest an implication of the approach advocated here for the understanding of individual differences in general. As many have pointed out in the past, we cannot deal constructively with individual differences when we identify the important variables as age, sex, grade, IQ, social status, and so on. The critical variables are process variables. The approach proposed here, the approach which makes individualdifferences variables crucibles in theory construction, will identify the process variables as a fallout from nomothetic theory construction if, of course, the nomothetic theories are dealing with fundamental processes of behavior.
Now that the article is completed, I find that I have exorcised the uneasy feelings that led to it. I had not hoped merely for therapy, but rather for a discussion of theory construction in the coming years. But even if this discussion does not ensue, you may be sure that the next student who asks me that question is likely to be severely imprinted.

\section{REFERENCES}

Broadbent, D. E. Perception and communication. New York: Pergamon Press, 1958.

Ekstrand, B. R., Wallace, W. P., \& Underwood, B. J. A frequency theory of verbal-discrimination learning. Psychological Review, 1966, 73, 566-578.

Gagné, R. M. (Ed.). Learning and individual differences. Columbus, Ohio: Charles E. Merrill, 1967.

\section{Publication of Milner and Kraft Addresses}

The Distinguished Scientific Contribution Award address, which Brenda Milner presented at the 1974 APA Convention in New Orleans, will appear in a subsequent issue of the American Psychologist. Conrad Kraft's Distinguished Contribution for Applications in Psychology Award address is also scheduled to appear in a future issue.

\section{Contributions to Education in Psychology Awards}

The American Psychological Foundation invites nominations for the 1975 Contributions to Education in Psychology Award. The annual award is in the amount of $\$ 1,000$ to be given to the recipient for his achievements. It is hoped that the awardee's institution will contribute a matching sum, thus providing the recipient with a modest "grant" to be used by him as he wishes to "improve the teaching of psychology." Thus, the objective of the award program is to enhance the local instructional program, not just to recognize "master teachers." Because the awards are to be presented at the APA Annual Convention in September 1975, nominations must be completed and sent to the Committee by March 31, 1975. A nomination form with a statement of the guidelines and suggestions for documentation can be obtained by writing to Edward $\mathrm{H}$. Loveland, Chairman, APF Teaching Awards Committee, Department of Psychology, Georgia Institute of Technology, Atlanta, Georgia 30332. 